\title{
THE THIRD IMAGE OF THE LARGE-SEPARATION LENSED QUASAR SDSS J1029+2623 ${ }^{1}$
}

\author{
Masamune Oguri, ${ }^{2}$ Eran O. OfeK, ${ }^{3}$ Naohisa Inada, ${ }^{4}$ Tomoki Morokuma, ${ }^{5}$ Emilio E. Falco, ${ }^{6}$ \\ Christopher S. Kochanek, ${ }^{7}$ Issha Kayo, ${ }^{8}$ Tom Broadhurst, ${ }^{9}$ and Gordon T. Richards ${ }^{10}$ \\ Received 2008 January 14; accepted 2008 January 31; published 2008 February 26
}

\begin{abstract}
We identify a third image in the unique quasar lens SDSS J1029+2623, the second known quasar lens produced by a massive cluster of galaxies. The spectrum of the third image shows similar emission and absorption features but has a redder continuum than the other two images, which can be explained by differential extinction or microlensing. We also identify several lensed arcs. Our observations suggest a complicated structure of the lens cluster at $z \approx 0.6$. We argue that the three lensed images are produced by a naked cusp on the basis of successful mass models, the distribution of cluster member galaxies, and the shapes and locations of the lensed arcs. Lensing by a naked cusp is quite rare among galaxy-scale lenses but is predicted to be common among large-separation lensed quasars. Thus the discovery can be viewed as support for an important theoretical prediction of the standard cold dark matter model.
\end{abstract}

Subject headings: galaxies: clusters: general — gravitational lensing quasars: individual (SDSS J102913.94+262317.9)

\section{INTRODUCTION}

SDSS J102913.94+262317.9 (SDSS J1029+2623; Inada et al. 2006) is one of two examples of strongly lensed quasars produced by massive clusters of galaxies. It was discovered in the Sloan Digital Sky Survey Quasar Lens Search (SQLS; Oguri et al. 2006, 2008; Inada et al. 2008), a survey to identify gravitationally lensed quasars from the spectroscopic sample of quasars in the Sloan Digital Sky Survey (SDSS; York et al. 2000). The image separation of $22.5^{\prime \prime}$ makes it the largest lensed quasar known to date. Inada et al. (2006) found that the system consists of two images of a radio-loud quasar at $z=2.197$ created by a massive cluster of galaxies at $z \sim 0.6$. The rareness of such quasar-cluster lens systems (e.g., Ofek et al. 2001; Phillips et al. 2001; Inada et al. 2003) validates the importance of understanding this system with extensive follow-up work.

In this Letter, we present new imaging and spectroscopic observations of this lens system. On the basis of these observations we identify a third lensed quasar image, measure the redshift of the lens cluster, and identify several additional lensed

\footnotetext{
${ }^{1}$ Some of the data presented herein were obtained at the W. M. Keck Observatory, which is operated as a scientific partnership among the California Institute of Technology, the University of California, and the National Aeronautics and Space Administration. The Observatory was made possible by the generous financial support of the W. M. Keck Foundation. This work is based in part on data collected at Subaru Telescope, which is operated by the National Astronomical Observatory of Japan. Use of the UH $2.2 \mathrm{~m}$ telescope for the observations is supported by NAOJ.

${ }^{2}$ Kavli Institute for Particle Astrophysics and Cosmology, Stanford University, 2575 Sand Hill Road, Menlo Park, CA 94025.

${ }^{3}$ Division of Physics, Mathematics and Astronomy, California Institute of Technology, Pasadena, CA 91125.

${ }^{4}$ Cosmic Radiation Laboratory, RIKEN (The Institute of Physical and Chemical Research), 2-1 Hirosawa, Wako, Saitama 351-0198, Japan.

${ }^{5}$ Optical and Infrared Astronomy Division, National Astronomical Observatory of Japan, Mitaka, Tokyo, 181-8588, Japan.

${ }^{6}$ Harvard-Smithsonian Center for Astrophysics, Cambridge, MA 02138.

${ }^{7}$ Department of Astronomy, Ohio State University, Columbus, OH 43210.

${ }^{8}$ Department of Physics and Astrophysics, Nagoya University, Chikusa-ku, Nagoya 464-8602, Japan.

${ }^{9}$ School of Physics and Astronomy, Tel Aviv University, Tel Aviv 69978, Israel.

${ }^{10}$ Department of Physics, Drexel University, 3141 Chestnut Street, Philadelphia, PA 19104.
}

arcs. We discuss the observations in $\S 2$, model and interpret the lens system in $\S 3$, and summarize our results in $\S 4$.

\section{FOLLOW-UP OBSERVATIONS}

We obtained deep images of the system using the University of Hawaii 2.2 m (UH88) and Keck I telescopes. In the UH88 observations we took $1000 \mathrm{~s} B$-band, $1200 \mathrm{~s}$ VRI-band, and $400 \mathrm{~s}$ VRI-band images on the nights of 2006 November 14, 2007 May 17, and 2007 November 12 using the Tektronix $2048 \times 2048$ CCD camera (Tek $2 \mathrm{k}$ ). The typical seeing was $\sim 0.8^{\prime \prime}$ and the conditions were photometric. The photometry was calibrated by the standard star PG 0918+029 (Landolt 1992). We obtained additional $720 \mathrm{~s} g$-band and $880 \mathrm{~s} R$-band images with the Keck I telescope using the Low Resolution Imaging Spectrometer with the Atmospheric Dispersion Compensator (LRIS-ADC; Oke et al. 1995) on 2008 January 4 when the seeing was $\sim 0.7^{\prime \prime}$. Since no standard star was observed, we calibrated the images using the UH88 and SDSS images. These data were reduced and analyzed using standard IRAF tasks.

We show the Keck image in Figure 1. The results of the photometry and astrometry are summarized in Table 1. Several lensed arcs are clearly visible in the image. As suggested in Inada et al. (2006), the central galaxy G1 consists of two components, which we name G1a and G1b. Inada et al. (2006) argued that object $\mathrm{C}$ is not a lensed image on the basis of its different color from images $\mathrm{A}$ and $\mathrm{B}$, and our follow-up results confirm that the color of object $\mathrm{C}$ is significantly different from those of the other lensed quasar images. However, both by comparing the UH88 $V$-band images and from an ongoing monitoring at the Fred Lawrence Whipple Observatory $1.2 \mathrm{~m}$ telescope (see Fohlmeister et al. 2007, 2008), we see that both B and $\mathrm{C}$ have faded by approximately $0.1 \mathrm{mag}$ during 2007 . This suggests that object $\mathrm{C}$ is a quasar, as variable blue sources at this magnitude level are typically quasars (e.g., Sesar et al. 2007).

We obtained spectra of objects B and C with the LRIS-ADC at the Keck I telescope on 2007 December 14 and spectra of galaxies G1a, G1b, and G2 with the Faint Object Camera and Spectrograph (FOCAS; Kashikawa et al. 2002) at the Subaru telescope on 2007 January 23. In the Keck LRIS-ADC observation, the $800 \mathrm{~s}$ exposure was taken with a long slit $\left(1.0^{\prime \prime}\right.$ 


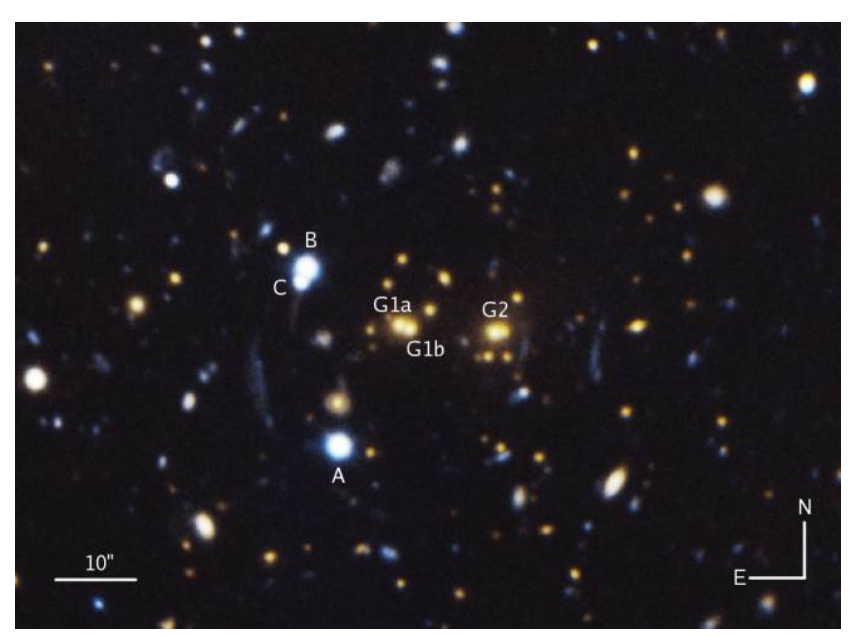

FIG. 1.-Follow-up color image of SDSS J1029+2623 produced from the Keck $g$ - and $R$-band images. The positions, magnitudes, and redshifts of the lensed images and cluster members are summarized in Table 1. Several lensed arcs are also seen. Note particularly two large blue arcs on the east and west sides of galaxies G1/G2, a red arc on the south of object C, and a blue arc located near galaxy G2.

width) aligned with objects B and C. We used the D560 dichroic with the 400/8500 grating and the 400/3500 grism to achieve a spectral resolution of $\sim 5 \AA$. The observations were obtained in nonphotometric conditions with poor $\sim 2.0^{\prime \prime}$ seeing. We used the deblending procedure of Pindor et al. (2006) in which we assumed Gaussian profiles for spatial cross sections to extract independent spectra of the two components. The standard star Feige 34 (Oke 1990) was used for flux calibration. The Subaru FOCAS observation was conducted in $2 \times 2$ on-chip binning mode with the 300B grism and the SY47 filter. The $900 \mathrm{~s}$ exposure was taken in $\sim 0.6^{\prime \prime}$ seeing, with a long slit (1.0" width) aligned across the galaxies G1a, G1b, and G2. The spectral resolution with this setup is $R \sim 400$.

Figure 2 unambiguously shows that component $\mathrm{C}$ is a quasar with the same redshift, $z_{s}=2.197$, as component B. The shapes of the emission and absorption lines are similar. Note in particular similar absorptions in the wings of the $\operatorname{Ly} \alpha, \mathrm{Si}$ IV, and $\mathrm{C}$ IV lines. These absorption features are probably intrinsic to the quasar and therefore serve as strong evidence that objects $\mathrm{B}$ and $\mathrm{C}$ are lensed images of the same quasar. As we expect from the different broadband colors, image $\mathrm{C}$ has a redder continuum than image B. A possible explanation is differential extinction, which is relatively common in lensed quasars (e.g.,
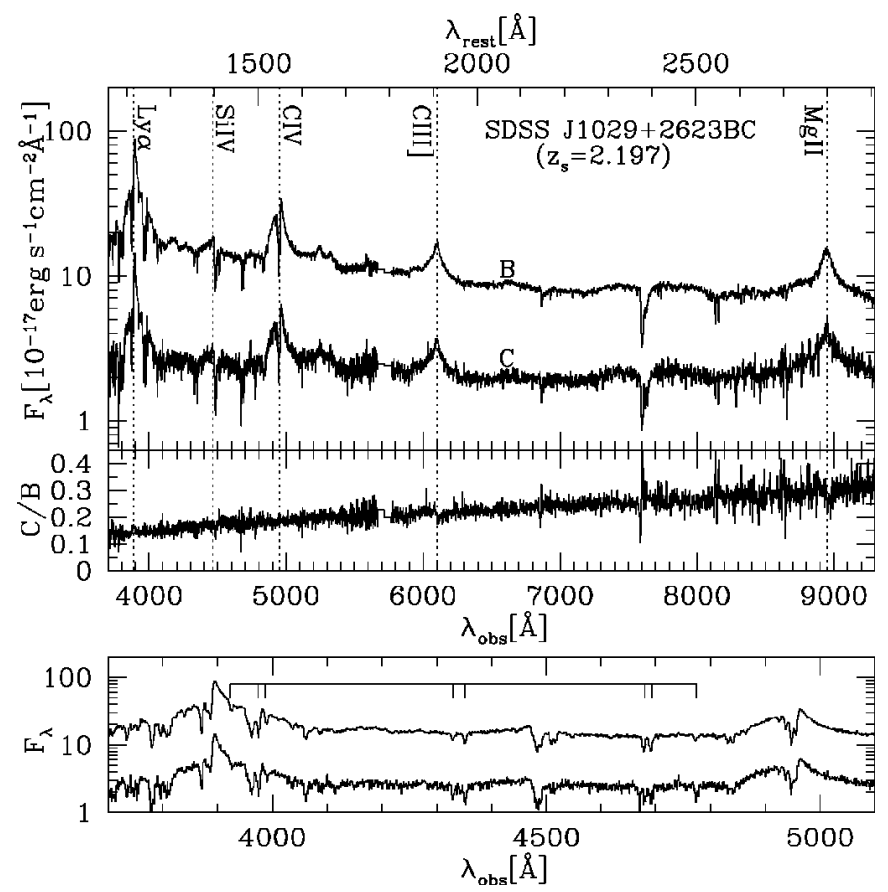

FIG. 2.-Top: Spectra of components B and C obtained with the LRIS-ADC at Keck. Quasar emission lines redshifted to $z_{s}=2.197$ are indicated by vertical dotted lines. The ratio of the spectra is also shown. Bottom: Expanded view of the blue side to show absorption systems more clearly. In addition to strong absorption lines associated with the emission lines, a $\mathrm{Mg}$ I/Mg II/Fe II absorption system at $z=0.674$, which is indicated by solid bars, is seen in both $\mathrm{B}$ and $\mathrm{C}$. We note that additional $\mathrm{Mg}$ II absorptions at $z=1.910$ (in $\mathrm{B}$ and $\mathrm{C}$ ) and $z=1.761$ (in $\mathrm{B}$ ) are also detected in the spectra.

Falco et al. 1999; Elíasdóttir et al. 2006). This requires that the two paths have $\Delta E(B-V) \sim 0.15-0.2$ assuming the extinction is caused by dust $\left(R_{V}=3.1\right)$ at the cluster redshift, $z \approx 0.6$. We subtracted models for images $\mathrm{B}$ and $\mathrm{C}$ from the Keck images and found no nearby galaxies beyond the red arc to the south of image C. Another possibility is microlensing by stars in the lens, which can also modify the color of the continuum (e.g., Poindexter et al. 2007; Ofek et al. 2007). We note that both dust reddening and microlensing are observed in the other cluster-scale lensed quasar, SDSS J1004+4112 (Oguri et al. 2004; Richards et al. 2004; Fohlmeister et al. 2007, 2008). Moreover, the Hubble Space Telescope image of this system also shows no obvious candidate galaxy for the effects. In principle, we can distinguish between these possibilities by examining the differences in the flux ratios between

TABLE 1

SDSS J1029+2623: Astrometry AND PhOtometry

\begin{tabular}{lrcccccccc}
\hline \hline Name & $\begin{array}{c}\Delta x \\
(\operatorname{arcsec})\end{array}$ & $\begin{array}{c}\Delta y \\
(\operatorname{arcsec})\end{array}$ & $B(\mathrm{UH})$ & $V(\mathrm{UH})$ & $R(\mathrm{UH})$ & $I(\mathrm{UH})$ & $g($ Keck $)$ & $R($ Keck $)$ & Redshift \\
\hline $\mathrm{A} \ldots \ldots \ldots$ & 0.00 & 0.00 & 19.20 & 18.72 & 18.55 & 18.01 & 18.72 & 18.46 & 2.197 \\
$\mathrm{~B} \ldots \ldots \ldots$ & -3.87 & 22.19 & 19.03 & 18.67 & 18.51 & 17.95 & 18.81 & 18.58 & 2.197 \\
$\mathrm{C} \ldots \ldots \ldots$ & -4.53 & 20.55 & 20.89 & 20.63 & 20.28 & 19.51 & 20.87 & 20.38 & 2.197 \\
$\mathrm{G} 1 \mathrm{\ldots} \ldots \ldots$ & 7.89 & 14.90 & $>23.5$ & 22.18 & 20.92 & 19.64 & 22.98 & 20.93 & 0.596 \\
$\mathrm{G} 1 \mathrm{~b} \ldots \ldots$ & 8.93 & 14.54 & $>23.5$ & 22.34 & 21.12 & 19.73 & 23.01 & 21.02 & 0.601 \\
$\mathrm{G} 2 \ldots \ldots$. & 19.85 & 14.00 & $>23.5$ & 22.00 & 20.62 & 19.26 & 22.69 & 20.60 & 0.584 \\
\hline
\end{tabular}

NotE. - The relative positions are from the UH88 I-band image. Note that the equatorial J2000.0 coordinates of object A are $10^{\mathrm{h}} 29^{\mathrm{m}} 13.94^{\mathrm{s}},+26^{\circ} 23^{\prime} 17.9^{\prime \prime}$. The UH88 images were taken on 2006 November $(B)$ and 2007 May (VRI), whereas the Keck images were obtained on 2008 January 4. The magnitudes are 3 " diameter aperture magnitudes. Measurement errors on the positions and magnitudes are typically $\leqslant 0.01^{\prime \prime}$ and $\lessgtr 0.02$ mag, respectively. All the redshifts are spectroscopic, from Inada et al. (2006) (A and B), Keck (B and C), and Subaru (G1a, G1b, G2). 
the continuum and the emission lines, because dust will affect both equally while microlensing affects the compact continuum emission region more strongly than the larger broad-line regions (e.g., Keeton et al. 2006). The ratio of the spectra shown in Figure 2 shows no strong difference between continuum and emission-line regions. However, we note a possible small difference in the $\mathrm{Mg}$ II emission line that suggests that microlensing is affecting this system.

Finally, the redshifts of G1a, G1b, and G2 are $z=0.596$, 0.601 , and 0.584 , respectively, confirming the estimated cluster redshift of $z \approx 0.6$. However, the velocity difference between G1b and G2 of $2800 \mathrm{~km} \mathrm{~s}^{-1}$ is so large that the system may be an ongoing merger or a superposition of two smaller clusters.

\section{INTERPRETING THE LENS SYSTEM}

Our identification of the third image has several important implications for the mass distribution of the lensing cluster. There are four possible explanations for image C: (i) it is a socalled central core image (Winn et al. 2004; Inada et al. 2005) of a "double" lens system; (ii) it is a "double" lens system, but one of the images is split into a pair of images because of external perturbations (e.g., Schechter \& Wambsganss 2002; Keeton 2003); (iii) the lens system is a standard cusp-type "quadruple" lens, but the fourth (counter) image is too faint to be observed; and (iv) the three images are produced by a naked cusp.

The main problem for $\mathrm{i}$ and ii is that the models require a large offset between the positions of the brightest cluster galaxies and the center of the lens potential, as discussed in Inada et al. (2006). In addition, the lensed arcs seem to contradict these scenarios as their shapes and locations suggest the lens potential is centered near G1 and G2 rather than somewhere between A and B. To test iii, we searched for any blue objects defined by $B-V<0.7, V-R<0.6$, and $R-I<1.0$ in the UH88 images. We expect the color cut is conservative as it includes image $\mathrm{C}$, which is significantly reddened. Except for the three confirmed lensed images, we found no such blue object, to the limiting magnitude of $B \sim 23.5$, within $60^{\prime \prime}$ of image A. This translates into a constraint on the flux ratio of the putative fourth image $\mathrm{D}$ of $D / A \lesssim 0.02$, which is strong enough to make scenario iii unlikely. We also searched for additional variable sources in the monitoring data and found none near the cluster, although with the present data we would not detect variability in a source significantly fainter than the known images.

The remaining scenario, iv, appears to be a viable solution. A naked cusp is a rare configuration of caustics in which a tangential caustic extends outside a radial caustic. In the case that a source is inside a naked cusp, three bright images are created on the same side of the potential center. For definiteness, we fit the positions of the three images using the lensmodel package (Keeton 2001). We adopt an elliptical Navarro et al. (1997) density profile centered on galaxy G1a with a fixed scale of $60^{\prime \prime}$. We note that the scale radius is degenerate with the estimated mass (Oguri et al. 2004). We assume a positional error of $0.5^{\prime \prime}$ to allow for perturbations from substructures (e.g., galaxies) in the cluster. This simple model, shown in Figure 3, works surprisingly well, with $\chi^{2}=8 \times 10^{-3}$ for 1 degree of freedom. The position angle of the model ( $-88^{\circ}$ east of north) is reasonable since it is aligned with the G1-G2 axis and the spatial distribution of color-selected cluster members and puts the lensed arcs on the major axis of the lens potential, as is

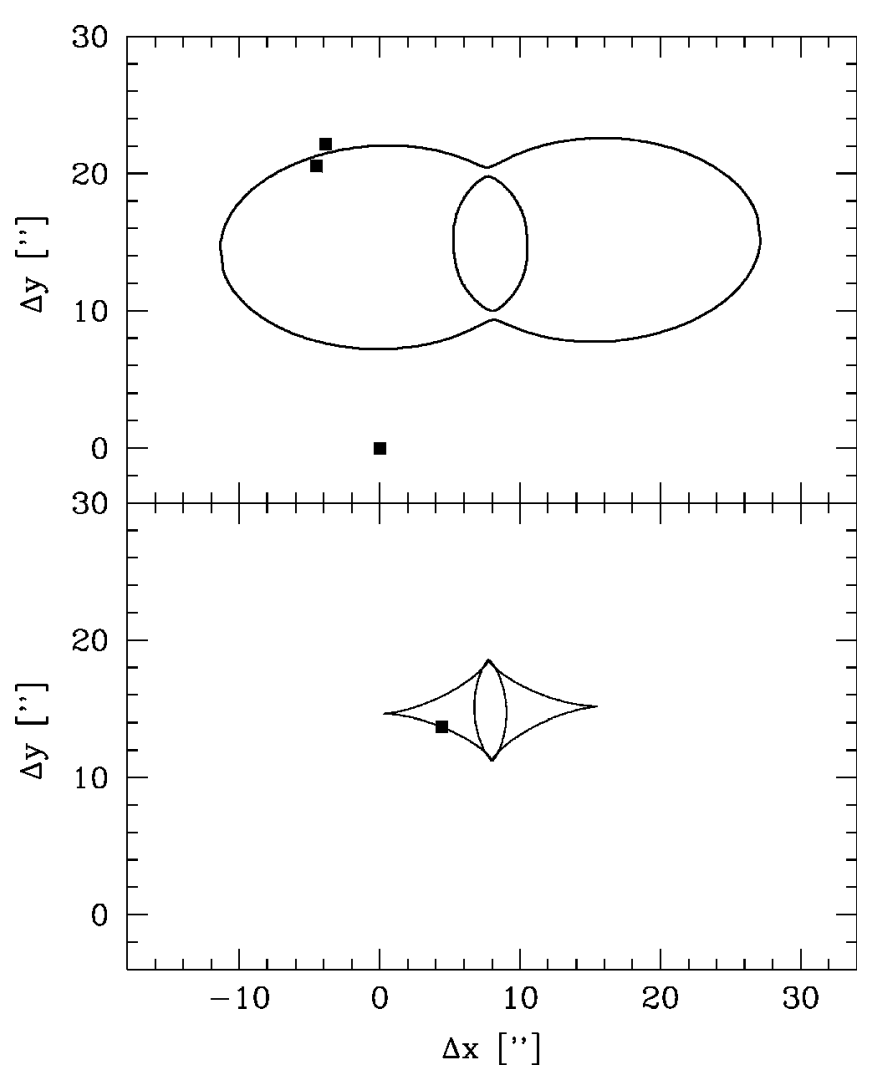

FIG. 3.-Critical curves (top) and caustics (bottom) of the best-fit model reproducing the three image positions. The square in the bottom panel shows the best-fit source position, whereas the three squares in the top panel indicate the corresponding best-fit image positions, which are very close to the observed image positions. Because of the naked cusp in the caustics, this model predicts only three images on the same side of the lens potential, which explains the unique image configuration of this lens system (see also Fig. 1). The model is an elliptical Navarro et al. (1997) profile with virial mass $M_{\text {vir }}=1.2 \times$ $10^{15} M_{\odot}$, concentration parameter $c_{\text {vir }}=4.9$, ellipticity $e=0.44$, and position angle $\theta_{e}=-88^{\circ}$. However, note that the derived mass and concentration parameter crucially depend on our assumption of the scale radius, $r_{s}=60^{\prime \prime}$.

preferred by ray-tracing in $N$-body simulations (Dalal et al. 2004). This model predicts time delays between the $A B$ and $\mathrm{BC}$ images of 1860 and $2.3 h^{-1}$ days, respectively, assuming a cosmological model of $\Omega_{M}=0.3$ and $\Omega_{\Lambda}=0.7$.

A caveat for this model, as in many models of gravitational lenses, is the flux ratios of the quasar images. Given the image geometry, we would generically expect $B \approx C>A$, while we observe $B \approx A>C$. Specifically, our model predicts $A: B: C=0.11: 1.00: 0.99$, whereas the observed flux ratios are $A: B: C=0.95: 1.00: 0.24$ in the UH88 I-band image. The strong wavelength dependence of the flux ratio between images $\mathrm{B}$ and $\mathrm{C}$, which is probably because of dust and/or microlensing, needs to be understood in order to fully evaluate this problem. We also note that the A/B flux ratio in the $6 \mathrm{~cm}$ Very Large Array (VLA) radio map of $\sim 0.73$ (Inada et al. 2006) is quite different from the ratio in the optical, $\sim 0.95$. The spatial resolution and signal-to-noise ratio of the radio map were not sufficient to derive robust radio fluxes for all the three components, and thus additional deep, high-resolution radio images will be essential for understanding the anomalous flux ratios.

\section{SUMMARY}

In Inada et al. (2006) we were left with a puzzle about SDSS J1029+2623: the observed geometry seemed inconsistent with 
the observed cluster center. Our discovery here that object $\mathrm{C}$ is a third image of the quasar, despite its redder continuum, solves that problem because a three-image cusp lens configuration is easily produced under these circumstances. The next problem is to understand the flux ratios of the system, both the wavelength dependences and that we observe $B \approx A>C$ rather than the expected $B \approx C>A$. Measuring the radio flux ratios will let us separate the effects of substructures in the cluster from extinction and microlensing, and the differences between continuum and emission line, radio flux ratios, and time variability will allow us to separate the effects of extinction and microlensing.

SDSS J1029+2623 is probably the second naked-cusp quasar lens. Among the 100 galaxy-scale lenses, only one candidate is known (APM 08279+5255; Lewis et al. 2002). Naked cusps require "marginal lenses," in which the lens has a surface density only moderately above the critical surface density required for the production of multiple images (see Blandford \& Kochanek 1987). The cooling of the baryons and the formation of the stellar component of a galaxy provides such high central surface densities that it is nearly impossible for a galaxy to have a naked cusp unless it is an edge-on disk-dominated system (see Keeton \& Kochanek 1998). Group and cluster halos, where the baryons have not cooled yet, are far less efficient lenses (e.g., Kochanek \& White 2001) and naked cusp configurations should be far more common. Specifically (see also Li et al. 2007; Minor \& Kaplinghat 2008), Oguri \& Keeton (2004) predicted that $30 \%-60 \%$ of cluster-scale lensed quasars will be naked-cusp systems depending on the inner density profile. ${ }^{11}$ We cannot seriously measure these fractions at present given one naked cusp lens and one conventional quadruple lens (SDSS J1004+4112; Inada et al. 2003), but the existence of even one naked-cusp cluster lens at this stage supports an important prediction of standard cold dark matter halos.

We thank Paul Schechter for useful discussions and an anonymous referee for helpful suggestions. This work was supported in part by Department of Energy contract DE-AC0276SF00515. C. S. K. is supported by NSF grant AST 07-08082. The authors wish to recognize and acknowledge the very significant cultural role and reverence that the summit of Mauna Kea has always had within the indigenous Hawaiian community. We are most fortunate to have the opportunity to conduct observations from this mountain.

\footnotetext{
${ }^{11}$ While cusp geometries also appear to be common for galaxies lensed by clusters, the more complex selection functions and the difficulty of identifying all the lensed images make it harder to do statistics.
}

\section{REFERENCES}

Blandford, R. D., \& Kochanek, C. S. 1987, ApJ, 321, 658

Dalal, N., Holder, G., \& Hennawi, J. F. 2004, ApJ, 609, 50

Elíasdóttir, Á., Hjorth, J., Toft, S., Burud, I., \& Paraficz, D. 2006, ApJS, 166, 443

Falco, E. E., et al. 1999, ApJ, 523, 617

Fohlmeister, J., Kochanek, C. S., Falco, E. E., Morgan, C. W., \& Wambsganss, J. 2008, ApJ, in press (arXiv:0710.1634)

Fohlmeister, J., et al. 2007, ApJ, 662, 62

Inada, N., et al. 2003, Nature, 426, 810

$$
\text { 2005, PASJ, 57, L7 }
$$

2006, ApJ, 653, L97

2008, AJ, 135, 496

Kashikawa, N., et al. 2002, PASJ, 54, 819

Keeton, C. R. 2001, preprint (astro-ph/0102340) 2003, ApJ, 584, 664

Keeton, C. R., Burles, S., Schechter, P. L., \& Wambsganss, J. 2006, ApJ, 639,1

Keeton, C. R., \& Kochanek, C. S. 1998, ApJ, 495, 157

Kochanek, C. S., \& White, M. 2001, ApJ, 559, 531

Landolt, A. U. 1992, AJ, 104, 340

Lewis, G. F., Carilli, C., Papadopoulos, P., \& Ivison, R. J. 2002, MNRAS, 330, L15
Li, G. L., Mao, S., Jing, Y. P., Lin, W. P., \& Oguri, M. 2007, MNRAS, 378, 469

Minor, Q. E., \& Kaplinghat, M. 2008, MNRAS, submitted (arXiv:0711.2537)

Navarro, J. F., Frenk, C. S., \& White, S. D. M. 1997, ApJ, 490, 493

Ofek, E. O., Maoz, D., Prada, F., Kolatt, T., \& Rix, H.-W. 2001, MNRAS, 324, 463

Ofek, E. O., Oguri, M., Jackson, N., Inada, N., \& Kayo, I. 2007, MNRAS, 382,412

Oguri, M., \& Keeton, C. R. 2004, ApJ, 610, 663

Oguri, M., et al. 2004, ApJ, 605, 78 2006, AJ, 132, 999 2008, AJ, 135, 512

Oke, J. B. 1990, AJ, 99, 1621

Oke, J. B., et al. 1995, PASP, 107, 375

Phillips, P. M., et al. 2001, MNRAS, 328, 1001

Pindor, B., et al. 2006, AJ, 131, 41

Poindexter, S., Morgan, N., Kochanek, C. S., \& Falco, E. E. 2007, ApJ, 660, 146

Richards, G. T., et al. 2004, ApJ, 610, 679

Schechter, P. L., \& Wambsganss, J. 2002, ApJ, 580, 685

Sesar, B., et al. 2007, AJ, 134, 2236

Winn, J. N., Rusin, D., \& Kochanek, C. S. 2004, Nature, 427, 613

York, D. G., et al. 2000, AJ, 120, 1579 